\title{
Prediction of spatiotemporal patterns of neural activity using a higher-order Markov representation of instantaneous pairwise maximum entropy model
}

\author{
Mehdi Aghagolzadeh', Karim Oweiss ${ }^{1,2^{*}}$ \\ From Twentieth Annual Computational Neuroscience Meeting: CNS*2011 \\ Stockholm, Sweden. 23-28 July 2011
}

Maximum entropy (MaxEnt) models have been suggested to fit the spatiotemporal patterns of simultaneously observed neuronal spike trains. To build such models, the distribution, $p_{x}(X)$, needs to be determined, where $X$ is a sequence of neural patterns formed from the vector $X^{1}, \ldots, X^{n}$ for $n$ time samples, where $X^{i}$ is a sample of the spike train of a population at time $i$. Each neural pattern itself is a binary sequence, for which one represents a spike, and zero otherwise. MaxEnt models are constructed by matching the moments between neuronal constituents with the analogous empirical estimates from the distribution $p_{x}(X)$.

MaxEnt models that incorporate the first and second order interactions among neurons, also referred to as the instantaneous pairwise MaxEnt model or the Ising model, are expressed as

$$
p\left(X^{t}\right)=\frac{1}{Z} \exp \left(-\sum_{i=1}^{N} h_{i} x_{i}^{t}-\frac{1}{2} \sum_{i=1}^{N} \sum_{j=1}^{N} J_{i j} x_{i}^{t} x_{j}^{t}\right)
$$

where the individual, $h_{i}$, and coupling, $J_{i j}$, terms are parameters associated with the first and second order interactions, respectively, and $Z$ is a normalization factor to insure $\sum_{x} p\left(X^{t}\right)=1$. This model was shown to be effective in representing almost 90 percent of the information in small populations (less than 20 neurons) [1,2]. The main disadvantage of this model, however, is that it

\footnotetext{
* Correspondence: koweiss@msu.edu

'Department of Electrical and Computer Engineering, Michigan State

University, East Lansing, MI, 48823, USA

Full list of author information is available at the end of the article
}

ignores the temporal correlation that represents the effect of the history of spiking activity on the current population state. Extending the width of neural patterns has been proposed as a method to incorporate spiking history. It destroys, however, the information in precise spike timings [3].

To incorporate the history term without compromising information in spike times, a higher-order Markov model is constructed in which patterns from different time samples are added. An example first-order Markov representation is expressed as

$$
\begin{aligned}
p\left(X^{t}, X^{t+1}\right)=\frac{1}{Z} \exp ( & -\sum_{i=1}^{N} h_{i}^{t} x_{i}^{t}-\frac{1}{2} \sum_{i=1}^{N} \sum_{j=1}^{N} J_{i j}^{t, t} x_{i}^{t} x_{j}^{t}-\sum_{i=1}^{N} \sum_{j=1}^{N} J_{i j}^{t, t+1} x_{i}^{t} x_{j}^{t+1} \\
& \left.-\sum_{i=1}^{N} h_{i}^{t+1} x_{i}^{t+1}-\frac{1}{2} \sum_{i=1}^{N} \sum_{j=1}^{N} J_{i j}^{t+1, t+1} x_{i}^{t+1} x_{j}^{t+1}\right)
\end{aligned}
$$

where $h_{i}^{t}$ and $h_{i}^{t+1}$ are the individual, and $J_{i j}^{t, t}, J_{i j}^{t, t+1}$ and $J_{i j}^{t+1, t+1}$ are the coupling terms among the neuronal elements. This spatiotemporal model is more general than the representation introduced in Marre et al [4] to account for history terms, but at the expense of increasing the number of parameters.

We evaluated the performance of the extended MaxEnt model in predicting the activity pattern of cat V1 neurons in response to drifting grating stimuli. A population of 21 neurons was recorded with 5 tetrodes. We compared the prediction power of this model with the instantaneous pairwise MaxEnt model, as well as the independent model. We show that the iterative scaling algorithm makes the extended model converge faster compared to [4], and also reduces the Kullback-Leibler 
divergence between the estimated and true distributions by 20 percent. Taken together, these results suggest the importance of accounting for temporal correlations in predicting the spatiotemporal patterns.

\section{Author details}

${ }^{1}$ Department of Electrical and Computer Engineering, Michigan State University, East Lansing, MI, 48823, USA. ${ }^{2}$ Neuroscience Program, Michigan

State University, East Lansing, MI, 48823, USA.

Published: 18 July 2011

\section{References}

1. Schneidman E, Berry MJ, Segev R, Bialek W: Weak pairwise correlations imply strongly correlated network states in a neural population. Nature 2006, 440:1012-1007.

2. Tang A, Jackson D, Hobbs J, Chen W, Smith JL, Patel H, Prieto A, Petrusca D, Grivich MI, Sher A: A maximum entropy model applied to spatial and temporal correlations from cortical networks in vitro. Journal of Neuroscience 2008, 28:505.

3. Truccolo W, Hochberg LR, Donoghue JP: Collective dynamics in human and monkey sensorimotor cortex: predicting single neuron spikes. Nature neuroscience 2009

4. Marre O, Boustani El S, Fregnac Y, Destexhe A: Prediction of spatiotemporal patterns of neural activity from pairwise correlations. Physical review letters 2009, 102:138101.

doi:10.1186/1471-2202-12-S1-P245

Cite this article as: Aghagolzadeh and Oweiss: Prediction of spatiotemporal patterns of neural activity using a higher-order Markov representation of instantaneous pairwise maximum entropy model. BMC Neuroscience 2011 12(Suppl 1):P245.

\section{Submit your next manuscript to BioMed Central} and take full advantage of:

- Convenient online submission

- Thorough peer review

- No space constraints or color figure charges

- Immediate publication on acceptance

- Inclusion in PubMed, CAS, Scopus and Google Scholar

- Research which is freely available for redistribution

Submit your manuscript at www.biomedcentral.com/submit
Ciomed Central 